\title{
The Mathematical Modeling of Metals Mass Transfer in Three Layer Peat Blocks
}

\author{
$\bar{E}$ rika Teirumnieka ${ }^{1,}$ Ilmārs Kangro ${ }^{1}$, Edmunds Teirumnieks $^{1,}$ Harijs Kalis $^{2}$ \\ 1 - Rezekne Higher Education Institution, Atbrīvošanas aleja 90, LV-4601, Rēzekne, Latvia, Tel. +371 \\ 4625150, Fax +3714625901 ; \\ e-mail: Erika.Teirumnieka@ru.lv,kangro@ru.lv,Edmunds.Teirumnieks@ru.lv \\ 2 - Institute of Mathematics, Latvian Academy of Sciences and University of Latvia, Zelllu 8, Riga LV - 1002, \\ Latvia, Tel.+37167033721, Fax+37167820113; e-mail: kalis@lu.lv
}

\begin{abstract}
The mathematical model for calculation of concentration of metals for 3 layers peat blocks is developed due to solving the 3-D boundary-value problem in multilayered domain - averaging and finite difference methods are considered.

As an example, mathematical models for calculation of Fe and Ca concentrations have been analyzed.
\end{abstract}

Keywords: averaging method, finite difference method, boundary conditions of third type, heavy metals, peat bog.

\section{INTRODUCTION}

Being based on the experimental data the mathematical model for calculation of concentration of metals in different points for different 3 layers (peat blocks) is developed.

An averaging [3] and finite difference method by solving the 3-D boundary-value problem with boundary conditions of the $3^{\text {rd }}$ type in the N-layered domain of homogeneous materials with piece-wise diffusion coefficients, the concentrations functions in every layer and the fixed source function are in question.

The boundary-value problem herein deals with solving the elliptic type of second order for the partial differential equation with piece-wise diffusion coefficients in the $\mathrm{N}$ layered domain.

Similarly, the results of a number of the previously modeled diffusion processes without mass transfer showed a comparatively good compatibility with the experimental data [1].

By using the averaging method with quadratic splines developed by A. Buikis [3], a finite-difference scheme was created. In its turn, it was used to reduce a 3-D boundary-value problem to a system of 2-D partial differential equations, which simplifies the task considerably.

\section{MATERIALS AND METHODS}

\section{A mathematical model}

The process of diffusion the metal in the peats block is consider in 3-D parallelepiped

$\Omega=\{(x, y, z): 0 \leq x \leq l, 0 \leq y \leq L, 0 \leq z \leq Z\}$

The domain - consist of multilauer medium. We will consider the stationary 3-D problem of the linear diffusion theory for multilayered piece-wise homogenous materials of $N$ layers in the form
$\Omega_{i}=\left\{(x, y, z): x \in(0, l), y \in(0 L), z \in\left(z_{i-1}, z_{i}\right)\right\}, i=\overline{1, N}$ where $H_{i}=z_{i}-z_{i-1}$ is the height of layer $\Omega_{i}$, $z_{0}=0, z_{N}=Z$. We will find the distribution of concentrations $c_{i}=c_{i}(x, y, z)$ in every layer $\Omega_{i}$ at the point $(x, y, z) \in \Omega_{i}$ by solving the following partial differential equation (PDE):

$D_{i x} \partial^{2} c_{i} / \partial x^{2}+D_{i y} \partial^{2} c_{i} / \partial y^{2}+D_{i z} \partial^{2} c_{i} / \partial z^{2}+f_{i}(x, y, z)=0($

where $D_{i x}, D_{i y}, D_{i z}$ are constant diffusions coefficients, $c_{i}=c_{i}(x, y, z)-$ the concentrations functions in every layer, $f_{i}(x, y, z)$ - the fixed sours function. The values $c_{i}$ and the flux functions $D_{i z} \partial c_{i} / \partial z$ must be continues on the contact lines between the layers $z=z_{i}, i=\overline{1, N-1}$ :

$\left.c_{i}\right|_{z_{i}}=\left.c_{i+1}\right|_{z_{i}}, D_{i z} \partial c_{i} /\left.\partial z\right|_{z_{i}}=D_{(i+1) z} \partial c_{i+1} /\left.\partial z\right|_{z_{i}}$,

$i=\overline{1, N-1}$

where $i=\overline{1, N-1}$.

We assume that the layered material is bounded above and below with the plane surfaces $z_{0}=0, z=Z$ with fixed boundary conditions in following form:

$$
c_{N}(x, y, Z)=C_{a}(x, y)
$$

where $C_{0}(x, y), C_{a}(x, y)$ are given concentrationfunctions, $\alpha$ is the mass transfer coefficient.

We have two forms of fixed boundary conditions in the $x, y$ directions: 
1) the periodical conditions by $x=0, x=l$ in the form

$c_{i}(0, y, z)=c_{i}(l, y, z), \partial c_{i}(0, y, z) / \partial x=\partial c_{i}(l, y, z) / \partial x$

2) the symetrical conditions by $y=0, y=L$

$\partial c_{i}(x, 0, z) / \partial y=\partial c_{i}(x, L, z) / \partial y=0$

For solving the problem (1.1)-(1.5) we will consider conservative averaging (AV) and finite difference (FD) methods. These procedures allow to reduce the 3-D problem to some $2 \mathrm{D}$ boundary value problem for the system of partial differential equations with circular matrix in the $x$-directions.

\section{The AV-method with quadratic splines}

The equation of (1.1) is averaged along the heights $H_{i}$ of layers $\Omega_{i}$ and quadratic integral splines along $z$ coordinate in following form one used [2]:

$$
\begin{aligned}
& c_{i}(x, y, z)=C_{i}(x, y)+m_{i}(x, y)\left(z-\bar{z}_{i}\right)+ \\
+ & e_{i}(x, y) G_{i}\left(\left(z-\bar{z}_{i}\right)^{2} / H_{i}^{2}-1 / 12\right)
\end{aligned}
$$

where $G_{i}=H_{i} / D_{i z}, \bar{z}_{i}=\left(z_{i-1}+z_{i}\right) / 2, m_{i}, e_{i}, C_{i}$ are the unknown coefficients of the spline-function, $C_{i}(x, y)=H_{i}^{-1} \int_{z_{i-1}}^{z_{i}} c_{i}(x, y, z) d z$ are the average values of $c_{i}, i=\overline{1, N}$.

After averaging the system (1.1) along every layer $\Omega_{i}$, we obtain system of $N$ equations of 2-D PDE

$D_{i x} \partial^{2} C_{i} / \partial x^{2}+D_{i y} \partial^{2} C_{i} / \partial y^{2}+2 H_{i}^{-1} e_{i}+F_{i}(x, y)=0$

where $F_{i}=H_{i}^{-1} \int_{z_{i-1}}^{z_{i}} f_{i}(x, y, z) d z$ are the average values of $f_{i}, i=\overline{1, N}$.

From boundary conditions (1.3) follows

$$
\begin{aligned}
& \frac{6}{\alpha}\left(D_{1 z} m_{1}-e_{1}\right)+3 m_{1} H_{1}=6\left(C_{1}-C_{0}\right)+e_{1} G_{1} \\
& 3 m_{N} H_{N}=6\left(C_{a}-C_{N}\right)-e_{N} G_{N}
\end{aligned}
$$

From (1.2) follows

$$
3 m_{i}+e_{i} G_{i}=6\left(C_{i+1}-C_{i}\right)-3 H_{i+1} m_{i+1}+e_{i+1} G_{i+1},
$$

$D_{i z} m_{i}+e_{i}=D_{(i+1) z} m_{i+1}-e_{i+1}, \quad i=\overline{1, N-1}$

From (2.1), (2.3), (2.4) we obtain following system of $N-2$ algebraic equations for determining $e_{i}$

$$
\begin{aligned}
& 2 e_{i-1} G_{i-1}\left(G_{i}+G_{i+1}\right)+e_{i}\left(\left(G_{i}+3 G_{i-1}\right)\left(G_{i}+G_{i+1}\right)+\left(G_{i}+3 G_{i+1}\right)\left(G_{i}+G_{i-1}\right)\right)+ \\
& +2 e_{i+1} G_{i+1}\left(G_{i}+G_{i-1}\right)=6\left(C_{i+1}-C_{i}\right)\left(G_{i}+G_{i-1}\right)-6\left(C_{i}-C_{i-1}\right)\left(G_{i}+G_{i+1}\right)
\end{aligned}
$$

and for determining $e_{1}, e_{N}$

$e_{1}\left(2 G_{1}+4 G_{2}+\frac{2}{\alpha}\left(4+\frac{6 G_{2}}{G_{1}}\right)\right)+2 e_{2}\left(G_{2}+\frac{2 G_{2}}{\alpha G_{1}}\right)=6\left(C_{2}-C_{1}\right)\left(1-\frac{2}{\alpha G_{1}}\right)$

$-6\left(C_{1}-C_{0}\right)\left(1+\frac{G_{2}}{G_{1}}\right)$

$e_{N}\left(2 G_{N}+4 G_{N-1}\right)+2 e_{N-1} G_{N-1}=-6\left(C_{N}-C_{N-1}\right)+$

$+6\left(C_{a}-C_{N}\right)\left(1+\frac{G_{N-1}}{G_{N}}\right)$

In the case $N=3$ (three layers) we have equations (2.6) and (2.5) for $i=2$.

Then

$e_{i}=e_{i, 1} C_{1}+e_{i, 2} C_{2}+e_{i, 3} C_{3}+e_{i, 0}$,

$m_{i}=m_{i, 1} C_{1}+m_{i, 2} C_{2}+m_{i, 3} C_{3}+e_{i, 0}, i=1 ; 2 ; 3$

From (2.2), (2.7) follows the system of three PDE

$$
\left\{\begin{array}{l}
D_{1 x} \partial^{2} C_{1}(x, y) / \partial x^{2}+D_{1 y} \partial^{2} C_{1}(x, y) / \partial y^{2}+ \\
+2 H_{1}^{-1} e_{1}(x, y)+\widehat{F}_{1}(x, y)=0 \\
D_{2 x} \partial^{2} C_{2}(x, y) / \partial x^{2}+D_{2 y} \partial^{2} C_{2}(x, y) / \partial y^{2}+ \\
+2 H_{2}^{-1} e_{2}(x, y)+\widehat{F}_{2}(x, y)=0 \\
D_{3 x} \partial^{2} C_{3}(x, y) / \partial x^{2}+D_{3 y} \partial^{2} C_{3}(x, y) / \partial y^{2}+ \\
+2 H_{3}^{-1} e_{3}(x, y)+\widehat{F}_{3}(x, y)=0
\end{array}\right.
$$

where ${ }^{(2.3)} F_{i}(x, y)=F_{i}(x, y)+2 H_{1}^{-1} e_{i, 0}, i=1 ; 2 ; 3$.

\section{The Finite Difference method}

For solving 2-D problems a uniform grid $\left(N_{x} \times\left(N_{y}+1\right)\right)$ is considered: 
$\omega_{h}=\left\{\begin{array}{l}\left(x_{i}, y_{j}\right), x_{i}=i h_{x}, y_{j}=(j-1) h_{y}, i=\overline{1, N_{x}}, \\ j=\overline{1, N_{y}+1}, N_{x} h_{x}=l, N_{y} h_{y}=L\end{array}\right\}$

Subscripts $(i, j)$ refer to $x, y$ indices, the mesh spacing in the $x_{i}, y_{j}$ directions are $h_{x}$ and $h_{y}$. The PDEs (2.7) can be rewritten in following vector form:

$D_{x} \partial^{2} C / \partial x^{2}+D_{y} \partial^{2} C / \partial y^{2}-A C+\widehat{F}=0$

where $D_{x}, D_{y}$ are the 3-d order diagonal matrices with elements $D_{1 x}, D_{2 x}, D_{3 x}$ and $D_{1 y}, D_{2 y}, D_{3 y}, C$ is the 3 order vectors-column with elements $C_{1}, C_{2}, C_{3}$, $\widehat{F}$ is also the vectors-column with elements $F_{1}, \widetilde{F}_{2}, \widetilde{F}_{3}$, and matrix $A$ is in following form:

$$
A=-2\left(\begin{array}{lll}
e_{1,1} / H_{1} & e_{1,2} / H_{1} & e_{1,3} / H_{1} \\
e_{21} / H_{2} & e_{22} / H_{2} & e_{23} / H_{3} \\
e_{3,1} / H_{3} & e_{3,2} / H_{3} & e_{3,3} / H_{3}
\end{array}\right) .
$$

The equation (3.1) with periodical conditions for vector function $C$ in the uniform grid $\left(x_{i}, y_{j}\right)$ is replaced by vector difference equations of second order approximation $[4,5]$.

They consist of vectors-columns and block-martices of order circulant symmetric matrixes.

The calculation of circulant matrix (matrix inversion and multiplication) can be carried out with MATLAB [2, 6]

The boundary conditions (1.5) are replaced by difference equations of first order approximation.

\section{The numerical methods}

The vectors-column $W_{j}$ of vector difference equations is calculated by Thomas algorithm in the matrix form using MATLAB.

$$
W_{j}=X_{j} W_{j+1}+Y_{j}=0, j=N_{y}(-1) 1
$$

where $X_{j}, Y_{j}$ are corresponding matrices and vectors, obtaining of following expressions

$$
\begin{aligned}
& X_{j}=\left(C C_{j}-A A_{j} X_{j-1}\right)^{-1} B B_{j} \\
& Y_{j}=\left(C C_{j}-A A_{j} X_{j-1}\right)^{-1}\left(A A_{j} Y_{j}+F_{j}\right), \quad j=2(1) N_{y}
\end{aligned}
$$

Here $X_{1}=E \quad Y_{1}=0 \quad W_{\widehat{N}+1}=\left(E-X_{\widehat{N}}\right)^{-1} Y_{\widehat{N}}$, $\left(\widehat{N}=N_{y}\right)$ where

$$
E=\left(\begin{array}{ccc}
{[1,0, \ldots, 0]} & 0 & 0 \\
0 & {[1,0, \ldots, 0]} & 0 \\
0 & 0 & {[1,0, \ldots, 0]}
\end{array}\right) .
$$

The inverse matrix of

$$
A=\left[\begin{array}{lll}
A 11 & A 12 & A 13 \\
A 21 & A 22 & A 23 \\
A 31 & A 32 & A 33
\end{array}\right]
$$

$B=A^{-1},(B A=A B=E)$ is in the form

$$
B=\left[\begin{array}{lll}
B 11 & B 12 & B 13 \\
B 21 & B 22 & B 23 \\
B 31 & B 32 & B 33
\end{array}\right]
$$

where $B$ is obtained due to MATLAB [6].

\section{Approbation of numerical algorithms}

We consider following test for the approbation of the calculations:

$f_{1}=f_{1}=f_{1}=C_{0}=0, \alpha=6 D_{1 z}, \alpha=600 D_{1 z}$, $C_{a}=C_{0 a} \cos (\pi y / L) \sin (2 \pi x / l), C_{0 a}=1$.

The special solutions in the form $c_{i}(x, y, z)=g_{i}(z) \cos (\pi y / L) \sin (2 \pi x / l), i=1 ; 2 ; 3$ of the PDE (1.1) can be obtain from following boundary value problem for three ODE (for conditions $(1.3,1.4)$ ):

$g_{1}^{\prime \prime}(z)-a_{1}^{2} g_{1}(z)=0$

$D_{1 z} g_{1}^{\prime}(0)-\alpha\left(g_{1}(0)-C_{0}\right)=0$,

$g_{2}^{\prime \prime}(z)-a_{2}^{2} g_{2}(z)=0, g_{3}^{\prime \prime}(z)-a_{3}^{2} g_{3}(z)=0$,

$g_{3}(Z)=C_{0 a}, g_{1}\left(H_{1}\right)=g_{2}\left(H_{1}\right)$,

$D_{1 z} g_{1}^{\prime}\left(H_{1}\right)=D_{2 z} g_{2}^{\prime}\left(H_{1}\right)$,

$g_{2}\left(L_{1}\right)=g_{3}\left(L_{1}\right), D_{2 z} g_{2}^{\prime}\left(L_{1}\right)=D_{3 z} g_{3}^{\prime}\left(L_{1}\right)$,

where $L_{1}=H_{1}+H_{2}, a_{i}=\pi \sqrt{\left(\frac{4 D_{i x}}{l^{2}}+\frac{D_{i y}}{L^{2}}\right) / D_{i z}}$,

$i=1 ; 2 ; 3$.

Therefore the exact solution is

$g_{1}(z)=P_{1} \sinh \left(a_{1} z\right)+P_{0} \cosh \left(a_{1} z\right)$,

$g_{2}(z)=P_{2} \sinh \left(a_{2} z\right)+P_{3} \cosh \left(a_{2} z\right)$

$g_{3}(z)=P_{4} \sinh \left(a_{3} z\right)+P_{5} \cosh \left(a_{3} z\right)$,

where coefficients $P_{i}, i=\overline{0,5}$ are functions dependent of $a_{i}, i=\overline{1,3}, D_{1 z}, D_{2 z}, D_{3 z}, \sinh (t), \cosh (t)$

(calculated for $t=a_{1} H_{1}, a_{2} H_{1}, a_{2} L_{1}, a_{3} L_{1}$ ), $\tanh (t), \operatorname{coth}(t)$ (calculated for $\left.t=a_{2} H_{1}, a_{3} L_{1}\right)$. 
The averaged values are

$C_{1}=H_{1}^{-1} \int_{0}^{H_{1}} g_{1}(z) d z=\frac{1}{a_{1} H_{1}}$

$\left(P_{1}\left(\cosh \left(a_{1} H_{1}\right)-1\right)+P_{0} \sinh \left(a_{1} H_{1}\right)\right)$

$C_{2}=H_{2}^{-1} \int_{H_{1}}^{L_{1}} g_{2}(z) d z=\frac{1}{a_{2} H_{2}}$

$\left(\begin{array}{l}P_{3}\left(\sinh \left(a_{2} L_{1}\right)-\sinh \left(a_{2} H_{1}\right)\right)+ \\ P_{2}\left(\cosh \left(a_{2} L_{1}\right)-\cosh \left(a_{2} H_{1}\right)\right)\end{array}\right)$

$C_{3}=H_{3}^{-1} \int_{L_{1}}^{Z} g_{3}(z) d z=\frac{1}{a_{3} H_{3}}$

$\left(\begin{array}{c}P_{5}\left(\sinh \left(a_{3} Z\right)-\sinh \left(a_{3} L_{1}\right)\right)+ \\ P_{4}\left(\cosh \left(a_{3} Z\right)-\cosh \left(a_{3} L_{1}\right)\right)\end{array}\right)$

We have following numerical results

$H_{1}=1, H_{2}=1.5, H_{3}=0.5, Z=$

$3.0, C_{0}=0, C_{0 a}=1$

for maximal and

minimal values of $c_{k}$ in the plane

$z=z_{k}, z_{k}=(k-1) h_{z}, k=\overline{1,12}, z_{13}=Z, z_{5}=H_{1}$,

$z_{11}=H_{1}+H_{2}, h_{z}=0.25$ by:
$D_{1 z}=10^{-3}, D_{2 z}=1.875 \cdot 10^{-3}$,

$D_{3 z}=0.1333 \cdot 10^{-3}, \alpha=600 D_{1 z}$ and $\alpha=6 D_{1 z}$,

$D_{1 x}=D_{1 y}=3 \cdot 10^{-3}, D_{2 x}=D_{2 y}=4 \cdot 10^{-4}$,

$D_{3 x}=D_{3 y}=5 \cdot 10^{-5}$

The numerical results by $N_{x}=N_{y}=20, N_{z}=12$ are given in the table $1\left(c_{a p}, c_{a n}\right.$ are the approximate and analytical-exact values). We have following averaged (integral) values:

1) $\alpha=600 D_{1 z} \quad$ for $C_{a p}: C_{1}=0.0646$,

$C_{2}=0.1894, C_{3}=0.6202$; for $C_{a n}: C_{1}=0.0663$,

$C_{2}=0.1931, C_{3}=0.6284$;

2) $\alpha=6 D_{1 z}$ - for $C_{a p}: C_{1}=0.1609, C_{2}=0.2545$,

$C_{3}=0.6482$; for $C_{a n}: C_{1}=0.1669, C_{2}=0.2617$, $C_{3}=0.6581$.

The numerical results for $N_{x}=N_{y}=40$ are coincided with 3 decimal places.

TABLE 1.

THE ANALYTICAL AND NUMERICAL RESULTS FOR 3 LAYERS (MAX. AND MIN. VALUES \pm )

\begin{tabular}{|l|l|l|l|l|}
\hline \multirow{2}{*}{$z_{k}$} & $\alpha=600$ & $\alpha=600$ & $\alpha=6$ & $\alpha=6$ \\
\cline { 2 - 5 } & $c_{a p}$ & $c_{a n}$ & $c_{a p}$ & $c_{a n}$ \\
\hline 0.00 & 0.0020 & 0.0021 & 0.1206 & 0.1257 \\
\hline 0.25 & 0.0328 & 0.0339 & 0.1395 & 0.1451 \\
\hline 0.50 & 0.0642 & 0.0659 & 0.1599 & 0.1659 \\
\hline 0.75 & 0.0963 & 0.0986 & 0.1820 & 0.1883 \\
\hline 1.00 & 0.1292 & 0.1321 & 0.2057 & 0.2123 \\
\hline 1.25 & 0.1476 & 0.1508 & 0.2196 & 0.2264 \\
\hline 1.50 & 0.1671 & 0.1705 & 0.2350 & 0.2420 \\
\hline 1.75 & 0.1877 & 0.1912 & 0.2521 & 0.2591 \\
\hline 2.00 & 0.2096 & 0.2133 & 0.2709 & 0.2780 \\
\hline 2.25 & 0.2331 & 0.2367 & 0.2917 & 0.2986 \\
\hline 2.50 & 0.2578 & 0.2617 & 0.3142 & 0.3213 \\
\hline 2.75 & 0.6189 & 0.6272 & 0.6469 & 0.6568 \\
\hline
\end{tabular}

III RESULTS AND DISCUSSION

6. The numerical results

6.1. Determining of the difussion coefficients in the $1 D$ case

In $1 \mathrm{D}$ case we have the boundary value problem of the following 3 ODEs

$$
\begin{aligned}
& c_{1}^{\prime \prime}(z)=0, c_{2}^{\prime \prime}(z)=0, c_{3}^{\prime \prime}(z)=0, \\
& D_{1 z} c_{1}^{\prime}(0)-\alpha\left(c_{1}(0)-C_{0}\right)=0, c_{3}(Z)=C_{0 a},
\end{aligned}
$$

$c_{1}\left(H_{1}\right)=c_{2}\left(H_{1}\right), D_{1 z} c_{1}^{\prime}\left(H_{1}\right)=D_{2 z} c_{2}^{\prime}\left(H_{1}\right)$,

$c_{2}\left(L_{1}\right)=c_{3}\left(L_{1}\right), \quad D_{2 z} c_{2}^{\prime}\left(L_{1}\right)=D_{3 z} c_{3}^{\prime}\left(L_{1}\right)$.

Using proportions $D_{1 z} / D_{2 z}=x_{1}, D_{1 z} / D_{3 z}=y_{1}$,

$D_{1 z} / \alpha=z_{1} z 1$ we have following solutions:

$c_{1}(z)=C_{1} z+C_{2}$,

$c_{2}(z)=C_{3} z+C_{4} c_{3}(z)=C_{5} z+C_{6}$,

where $C_{1}=\frac{C_{0 a}-C_{0}}{H_{1}+x_{1} H_{2}+y_{1} H_{3}+z_{1}}$,

$C_{2}=C_{0}+z_{1} C_{1}, C_{3}=x_{1} C_{1}$, 
$C_{4}=C_{0 a}-C_{1}\left(L_{1} x_{1}+y_{1} H_{3}\right), C_{5}=y_{1} C_{1}$,

$C_{6}=C_{0 a}-C_{1} y_{1} Z$.

From experimentally obtained data $c_{1}(0)=C_{00}$, $c_{1}\left(H_{1}\right)=C_{01}, c_{2}\left(L_{1}\right)=C_{02}$, we can determin the relations

$x_{1}=\frac{H_{1}\left(C_{02}-C_{01}\right)}{H_{2}\left(C_{01}-C_{00}\right)}, y_{1}=\frac{H_{1}\left(C_{a}-C_{02}\right)}{H_{3}\left(C_{01}-C_{00}\right)}$,

$z_{1}=\frac{H_{1}\left(C_{00}-C_{0}\right)}{\left(C_{01}-C_{00}\right)}$.

We consider the data for 2 metals in the peat blocks with $H_{1}=1, H_{2}=1.5, H_{3}=0.5$ :

1) for Fe: $C_{0 a}=0.188, C_{0}=0, C_{00}=0.66$,

$C_{01}=0.83, C_{02}=1.50$ we get obtain

$D_{2 z}=0.38 D_{1 z}, D_{3 z}=0.22 D_{1 z}, \alpha=0.26 D_{1 z}$,

2) for Ca: $C_{0 a}=4.63, C_{0}=0, C_{00}=1.30$,

$C_{01}=1.90, C_{02}=2.38$ we get obtain

$D_{2 z}=1.875 D_{1 z}, D_{3 z}=0.133 D_{1 z}, \alpha=0.46 D_{1 z}$.

We obtain with MAPLE by $D_{1 z}=10^{-3}$ the figures (Fig.

$1,2)$ and the coefficients

$C_{1}, C_{2}, C_{3}, C_{4}, C_{5}, C_{6}$ depending of $C_{00}, C_{01}, C_{02}$.

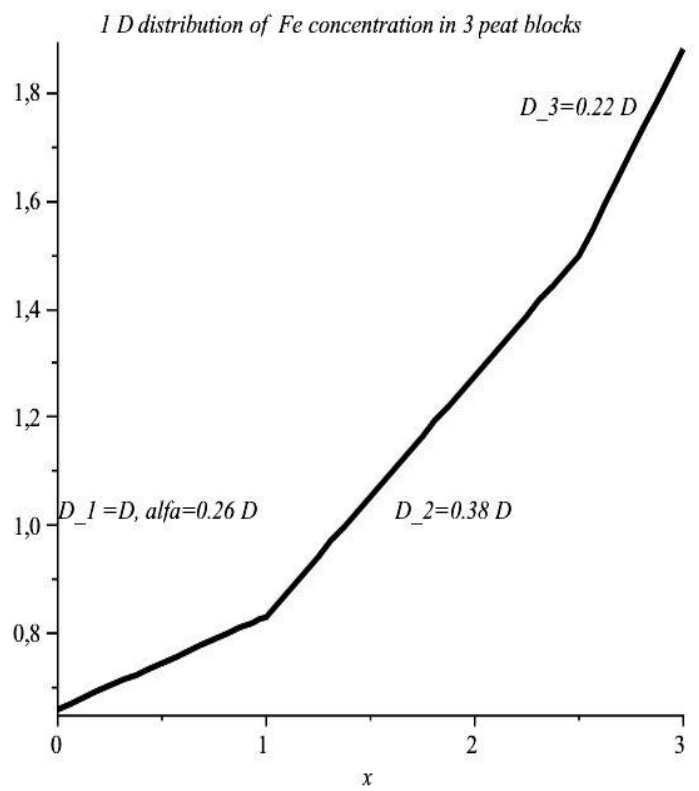

Fig. 1. Fe distribution and $D_{z}$

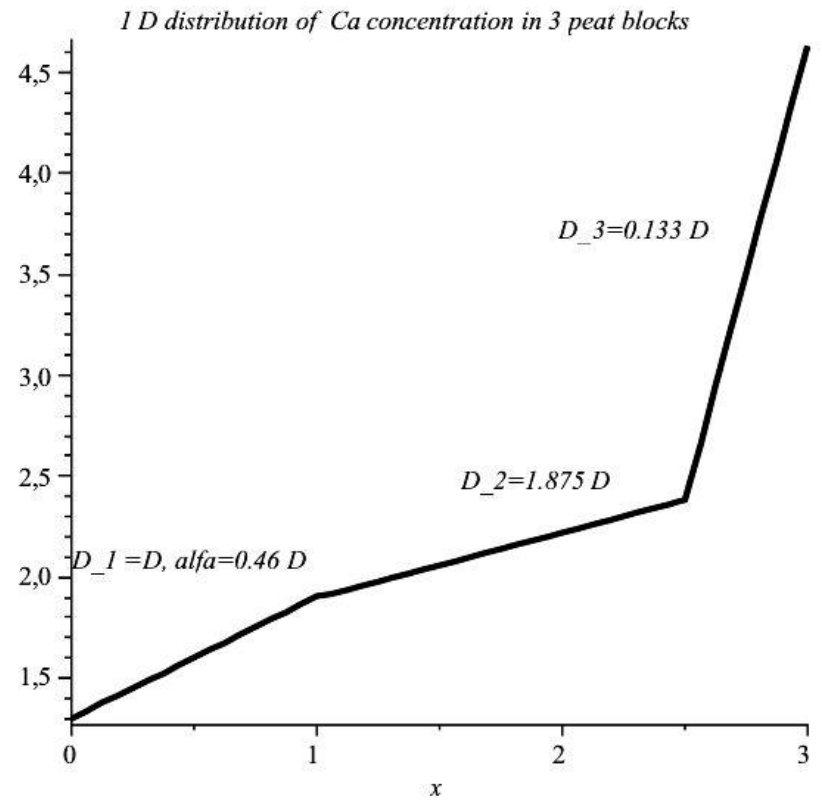

Fig. 2. Ca distribution and $D_{z}$

6.2. The calculation of concentration of metals in peat blocks

We consider the metals $\mathrm{Fe}$ and $\mathrm{Ca}$ concentration in the 3 layered peat blocks $\Omega$ with $L=l=1 \mathrm{~m}^{\text {- dimensions of }}$ the block's base, $H_{1}=1 \mathrm{~m}, H_{2}=1.5 \mathrm{~m}$,

$H_{3}=0.5 m^{\prime} L_{1}=H_{1}+H_{2}=2.5 m^{\prime}$

$Z=H_{1}+H_{2}+H_{3}=3 m$ - heights of the layers.

On the top of the earth $(z=Z)$ we have the measured concentration $c \mathrm{mg} / \mathrm{kg}$ of metals in the following nine points in the $(x ; y)$ plane:

1) for $\mathrm{Fe}: c(01 ., 0.2)=1.69 ; c(0.5,0.2)=1.83 ; c(0.9$, $0.2)=1.72 ; \quad c(0.1,0.5)=1.70 ; c(0.5,0.5)=1.88 ; c(0.9$, $0.5)=1.71 ; \quad c(0.1,0.8)=1.71 ; c(0.5,0.8)=1.82 ; c(0.9$, $0.8)=1.73$

2) for $\mathrm{Ca}$ :

$c(0.1,0.2)=3.69 ; c(0.5,0.2)=4.43 ; c(0.9,0.2)=3.72$; $c(0.1,0.5)=4.00 ; c(0.5,0.5)=4.63 ; c(0.9,0.5)=4.11$; $c(0.1,0.8)=3.71 ; c(0.5,0.8)=4.50 ; c(0.9,0.8)=3.73$.

This date are smoothing by $2 \mathrm{D}$ interpolation with MATLAB operator, using the spline function.

We have following diffusion coefficients in the layers:

1) for $\mathrm{Fe}:\left(C_{00}=0.66, C_{0 a}=1.88\right)$ :

$D_{1 z}=10^{-3}, D_{2 z}=0.38 \cdot 10^{-3}, D_{3 z}=0.22 \cdot 10^{-3}$,

2) for $C a:\left(C_{00}=1.30, C_{0 a}=4.63\right)$ :

$$
\begin{aligned}
& D_{1 z}=10^{-3}, \quad D_{2 z}=1.875 \cdot 10^{-3} \\
& D_{3 z}=0.1333 \cdot 10^{-3},
\end{aligned}
$$


Teirumnieka Ē., Kangro I., Teirumnieks E., Kalis H. THE MATHEMATICAL MODELING OF METALS MASS TRANSFER IN THREE LAYER PEAT BLOCKS

The diffusion coefficients in $x, y$ directions are:

$D_{1 x}=D_{1 y}=3 \cdot 10^{-4}, D_{2 x}=D_{2 y}=4 \cdot 10^{-4}$, $D_{3 x}=D_{3 y}=5 \cdot 10^{-5}$.//

In the Fig. 3, Fig. 4 graphics of metals concentration $c$ depending of vertical coordinate $z$ by $x=l / 2, y=L / 2$ and in other points are given.

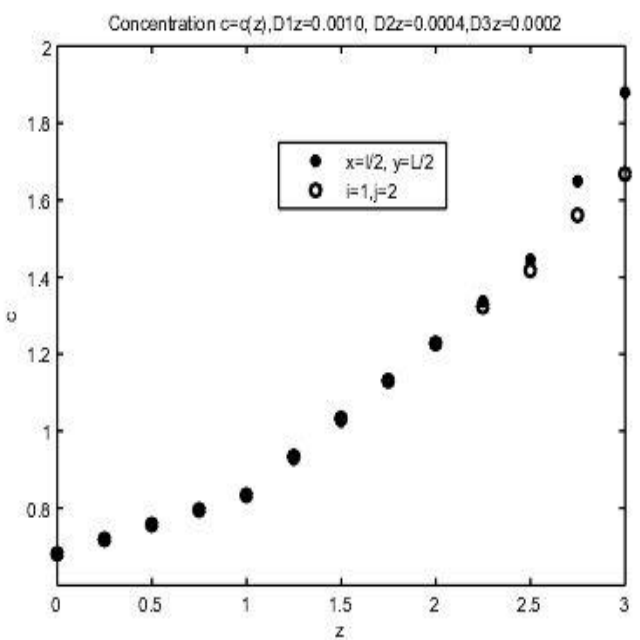

Fig. 3. Fe concentration $c$ depending on $\mathrm{z}$

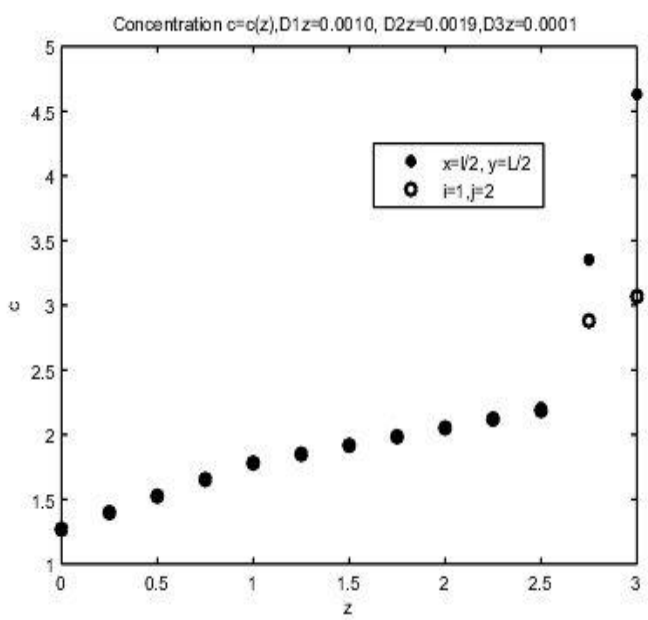

Fig. 4. Ca concentration $c$ depending on $\mathrm{z}$

The distribution of concentration $c$ in the $(x, y)$ plane is given: by $z=H_{1}$ (Fig. 5, 6.), $z=L_{1}=H_{1}+H_{2}$ (Fig. 7, 8.) $Z=H_{1}+H_{2}+H_{3}$ (Fig. 9, 10.).

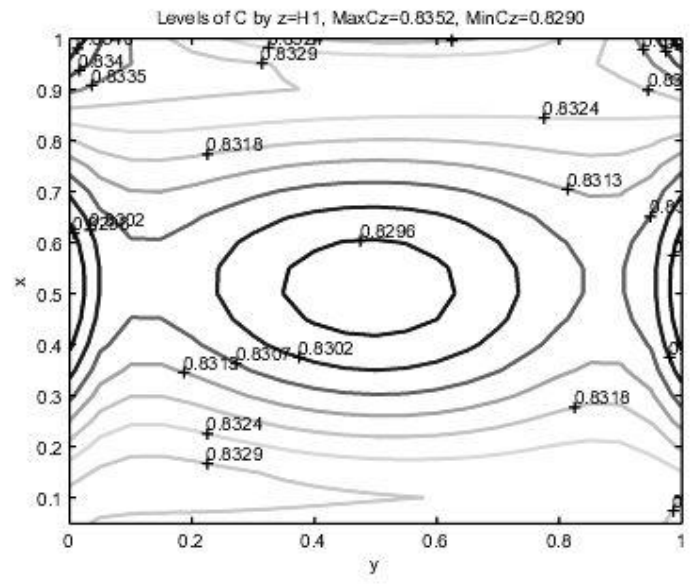

Fig. 5. Fe concentration $c$ in the $(\mathrm{x} ; \mathrm{y})$ plane by $\mathrm{z}=\mathrm{H} 1$

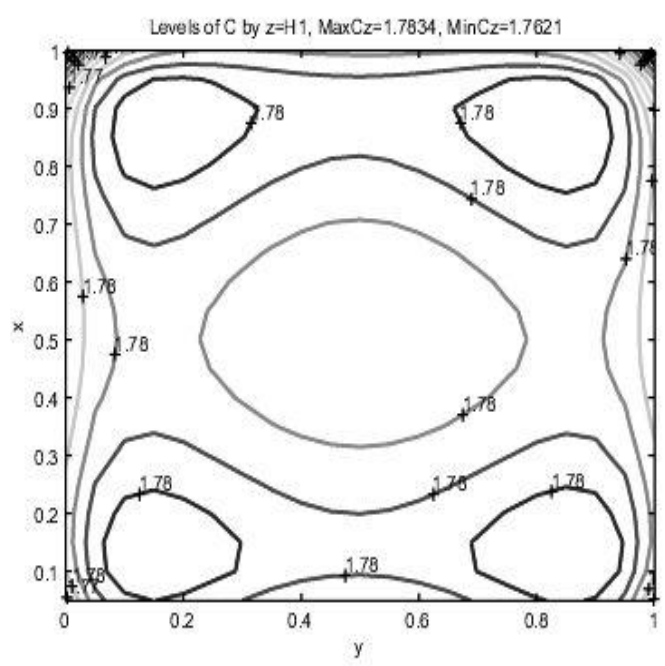

Fig.6. Ca concentration $c$ in the $(\mathrm{x} ; \mathrm{y})$ plane by $\mathrm{z}=\mathrm{H} 1$

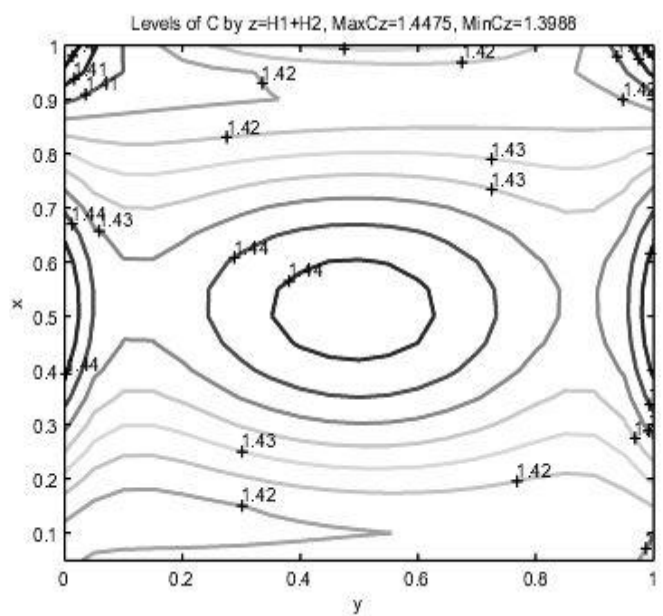

Fig. 7. Fe concentration $c$ in the $(\mathrm{x} ; \mathrm{y})$ plane by $\mathrm{z}=\mathrm{H} 1+\mathrm{H} 2$ 
Teirumnieka E., Kangro I., Teirumnieks E., Kalis H. THE MATHEMATICAL MODELING OF METALS MASS TRANSFER IN THREE LAYER PEAT BLOCKS

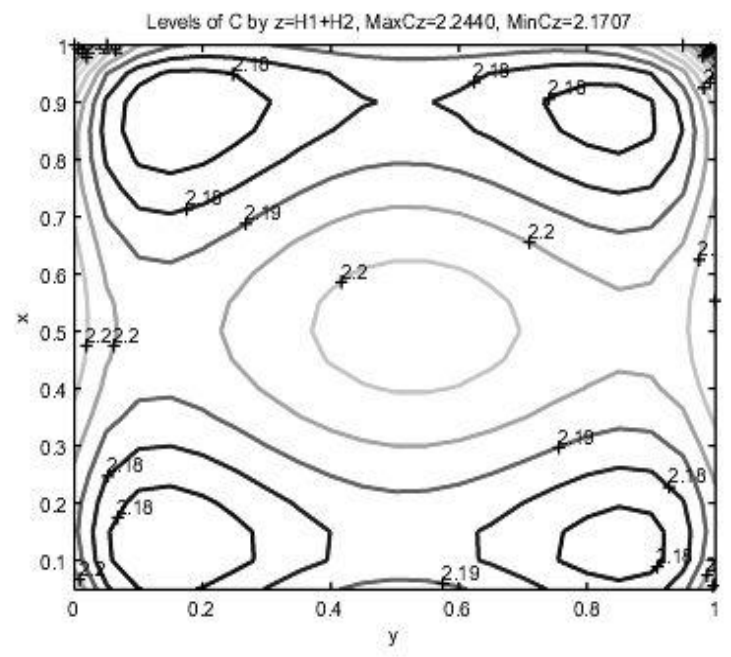

Fig. 8. Ca concentration $c$ in the (x;y) plane by $\mathrm{z}=\mathrm{H} 1+\mathrm{H} 2$

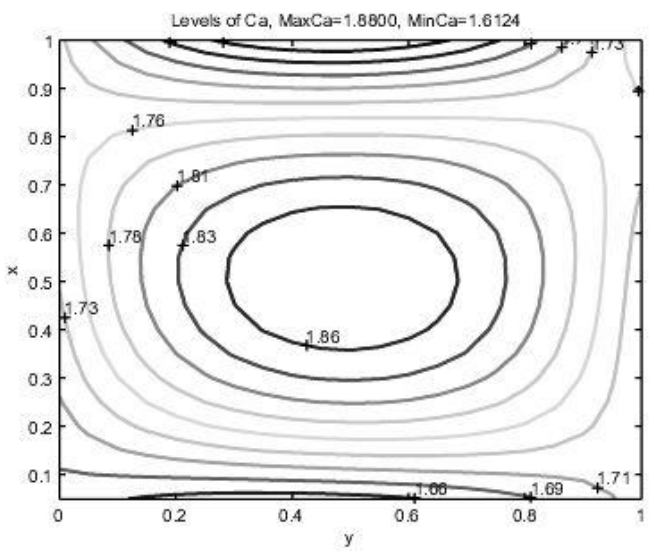

Fig. 9. Fe concentration $c$ in the (x;y) plane by $\mathrm{z}=\mathrm{Z}$

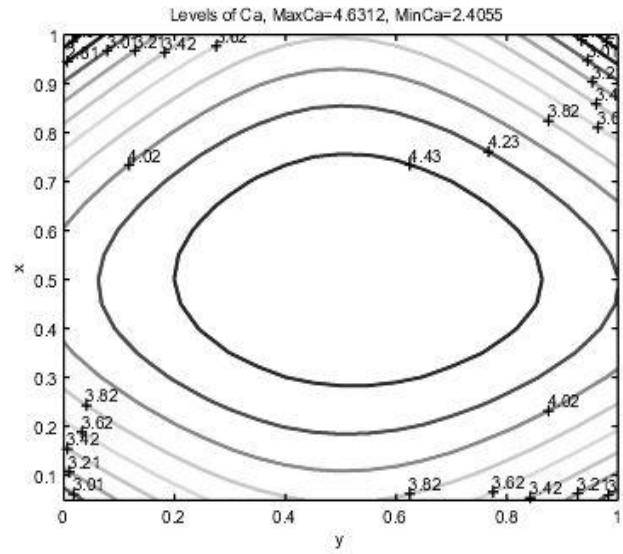

Fig. 10. Ca concentration $c$ in the (x;y) plane by $\mathrm{z}=\mathrm{Z}$
The distribution of concentration $c$ in the $(z, x)$ plane by $y=L / 2$ accordingly for $\mathrm{Fe}$ and $\mathrm{Ca}$ is given in Fig. 11, Fig. 12.

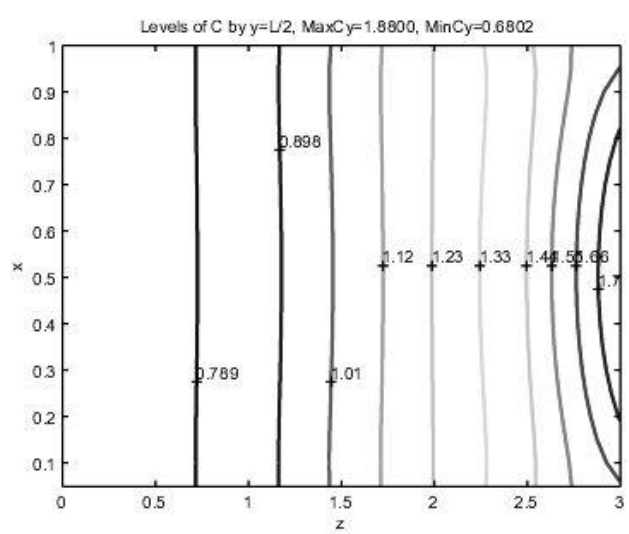

Fig. 11. Fe concentration $c$ depending of vertical coordinate $\mathrm{z}$ by $\mathrm{y}=\mathrm{L} / 2$

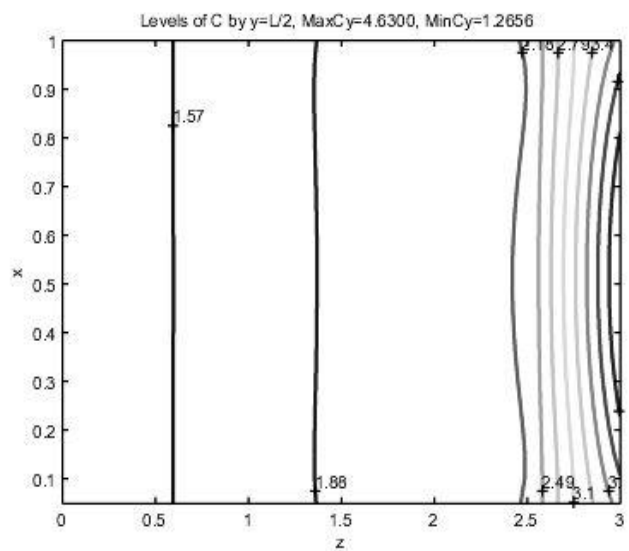

Fig. 12. Ca concentration $c$ depending of vertical coordinate $\mathrm{z}$ by $\mathrm{y}=\mathrm{L} / 2$

And the averaged values of concentration $c$ in the second layer are given in Fig. 13, Fig. 14.

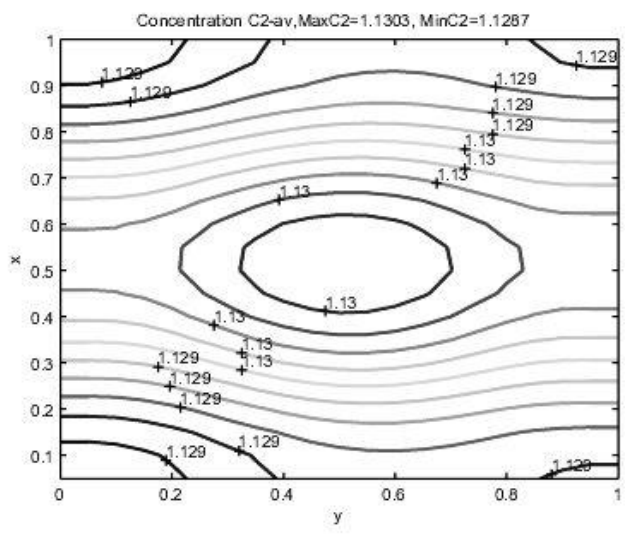

Fig. 13. Averaged values $\mathrm{C} 2$ in second layer for Fe concentration 


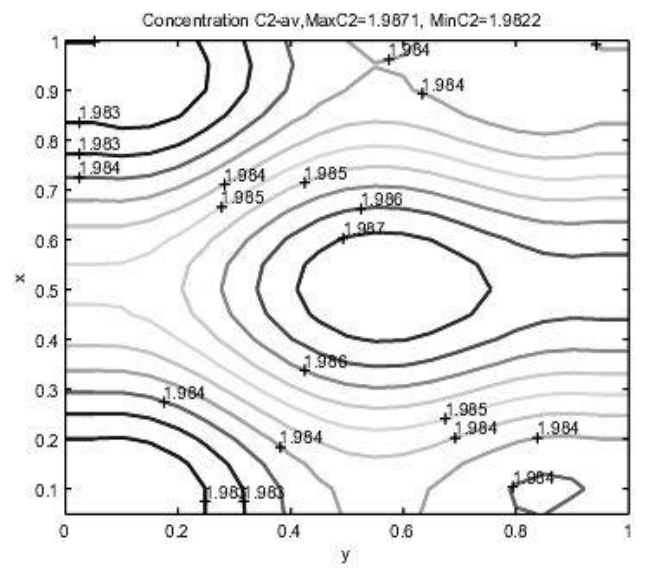

Fig. 14. Averaged values $\mathrm{C} 2$ in second layer for Ca concentration

\section{CONCLUSION}

The 3D diffusion problem in $N$ layered domain described by a boundary value problem of the system of PDEs with piece-wise constant diffusion coefficients are approximate on the 2D boundary value problem of a system of $N$ PDEs.

As opposed to the models analyzed previously $[1,4]$, the newly established mathematical model envisages modeling mass transfer in N-layers, and the boundary conditions of the $3^{\text {rd }}$ type included in the model enable the modeling of the substance flux through the boundary surface of the specified area in the direction of Earth's interior.
Test samples with an analytical solution (the indicator of transfer process) were developed and a numerical experiment was used to test and analyze the established method for three layers in comparison with the methods described previously.

The analytical and numerical results were coincided with 3 decimal places and it means that mathematical model have a practical application in real determination of trace elements concentrations.

The established mathematical model is applicable in studying transfer processes, where substance mass is transferred through boundary surfaces of the specified area, for instance, in a purification plant, purifying works etc.

\section{REFERENCES}

[1] Aboltinsh, A. (1983). The mathematical description of the sorption process. In.: Прикладные задачи математической физики. Сб. Научных Тр. Рига ЛГУ им. П. Стучки, 1983, с. 3-12. (in Russian).

[2] Bear, J. Hydraulic of groundwater. Mc.Graw-Hill Inc.,1979.

[3] Buikis, A. The approximation with splines for problems in layered systems. Riga, Acta Universitatis Latviensis, 592 (1994) 135-138 (in Latvian).

[4] Kangro, I., Gedroics, A., Kalis, H. (2011). About mathematical modelling of peat blovks in 3-layered 3D domain. In.: Abstracts of the Int. conf.: MATHEMATICAL MODELLING AND ANALYSIS May 25 - 28, 2011 Sigulda, Latvia, p.67.

[5] Teirumnieka, E., I. Kangro, I., Teirumnieks, E., Kalis, H., Gedroics, A. The mathematical modeling of $\mathrm{Ca}$ and $\mathrm{Fe}$ distribution in peat layers. Proc. of the 8-th int. Scientific and Practical Conference "Environment. Technology. Resources. June 20-22, 2011. 'Rezekne Higher Education institution, 2011, pp. 40-47.

[6] Thomas, J. W., Numerical partial differential equations. Finite difference methods. Springer- Verlag, New-York, Inc.,1995. 